\title{
Treating Graves' orbitopathy: where are we?
}

\author{
Maria Laura Tanda $\cdot$ Eliana Piantanida • \\ Luigi Bartalena
}

Published online: 25 January 2012

(C) Springer Science+Business Media, LLC 2012

Medical management of overt and active Graves' orbitopathy is frequently unsatisfactory, both for the patient and the physician [1]. Systemic glucocorticoids, more efficaciously given intravenously [2], represent the firstline treatment $[3,4]$, but many patients require a second course of steroids, associated with orbital radiotherapy or cyclosporine, to inactivate the disease [5]. And, eventually, some kind of rehabilitative surgery (orbital decompression, squint surgery, eyelid surgery) is needed in many patients when GO is inactive to correct residual invalidating manifestations, such as exophthalmos, strabismus/diplopia, eyelid retraction [3].

Reasons why glucocorticoids are not always effective are not fully understood. They include selection of patients with long-lasting GO, prevalence of proptosis with limited inflammatory changes, long-standing eye muscle dysfunction associated with fibrotic changes, smoking habits, mutations in the glucocorticoid receptor causing a decreased sensitivity to the drug. Most importantly, glucocorticoids, despite immunosuppressive actions, are mainly anti-inflammatory drugs and cannot be really considered pathogenic drugs. Autoimmune pathogenesis of GO is extremely complex, involving autoreactive T lymphocytes, B-lymphocytes, the TSH receptor, the IGF-1 receptor, secretion of a number of cytokines automaintaining the reactions occurring in the orbit. Studies are ongoing to completely unveil the multiple steps of the cascade of events eventually causing remodeling of the

M. L. Tanda $\cdot$ E. Piantanida $\cdot$ L. Bartalena $(\square)$ Department of Clinical \& Experimental Medicine, University of Insubria, Endocrine Unit, Ospedale di Circolo, Viale Borri, 57, 21100 Varese, Italy

e-mail: luigi.bartalena@uninsubria.it orbital tissue, with an increase in the fibroadipose tissue and swelling of the extraocular muscles [6]. These studies are essential to define the target of novel ("pathogenic") treatments (T-cells? B-cells? Cytokines?). Among clinical studies based on better understanding of orbital pathophysiological processes, promising, although preliminary, results come from the evaluation of the $\mathrm{CD} 20+$ depleting agent, rituximab [7] or some anti-TNF $\alpha$ agents [8]. In patients with mild GO, selenium administration for 6 months prevented the progression to more severe forms of eye disease [9]. Expansion of the orbital adipose tissue is undoubtedly a key feature of GO under most circumstances. In this issue of Endocrine, Zhang et al. [10] evaluated the effects of thalidomide on preadipocytes (3T3-L1 preadipocytes) and orbital fibroblasts from few patients with GO. This agent was initially used as an oral sedative hypnotic, but it is now used in the management of several autoimmune disorders in view of its anti-inflammatory and anti-angiogenic features. In Zhang et al.'s study, thalidomide dose-dependently inhibited adipogenesis of 3T3-L1 preadipocytes; this was associated with a decreased expression of PPAR $\gamma$ and the TSH receptor [10]. These inhibitory effects were also observed on the differentiation of preadipocyte fibroblasts from GO orbital tissue into adipocytes, in which the expression of TNF $\alpha$ and IL-6 was also reduced by thalidomide [10]. The main limitation of this ex vivo study is the small size, because tissue from only three patients with GO was utilized. However, it seems reasonable to try and reproduce these preliminary results in larger studies before envisioning or advocating its possible use in clinical practice in patients with GO. Pathogenesis of GO is so complex and implies the participation of so many actors that it is presently hard to believe that a single drug can cure or control the disease. It is however worth keep on trying. 
Another relevant and unsolved issue in this field pertains to the identification of the optimal treatment for hyperthyroidism in patients with GO [11]. Some authors prefer to treat GO and maintain patients euthyroid under longterm antithyroid drug treatment; others support the idea of thyroid ablation (preferably early thyroid ablation) to interrupt the link between the thyroid and the orbit [11]. The superiority of either approach is unsettled. In a retrospective study GO had a more favorable course, if, after thyroidectomy, patients were submitted to thyroid remnant ablation by radioiodine for incidentally found thyroid carcinoma [12]. In a more recent randomized clinical trial, a better outcome of mild-to-moderate and active GO treated with intravenous glucocorticoids and orbital radiotherapy was observed in a short-term follow-up in patients treated by total thyroid ablation (thyroidectomy followed by radioiodine ablation) than in those treated by surgery alone [13]. In this issue of Endocrine, De Bellis et al. [14] evaluated a series of 60 consecutive patients with mild-tomoderate GO of relatively recent onset, who were treated either by surgery alone, surgery followed by radioiodine therapy (because of incidental thyroid carcinoma), or by antithyroid drugs. The authors found that both surgery and total thyroid ablation were associated with an earlier and more pronounced amelioration of GO compared to antithyroid drug treatment [14]. This different trend in the outcome of GO was accompanied by an earlier disappearance of GO-related autoantibodies (TSH-receptor and G2s antibodies) [14]. This study bears limitations related to its non-randomized nature: (i) Patients in the first two groups underwent thyroid surgery because of large goiters, while thyroid size was much smaller in patients receiving antithyroid drugs; (ii) The number of patients given radioiodine after thyroidectomy was much smaller than that of patients treated by thyroidectomy alone (10 vs. 25). However, this study further underscores the importance of the thyroid-eye link and the potential benefit from breaking this link by thyroid ablation. Particularly if performed early in the course of the disease [15]. Admittedly, a conclusive answer to the superiority of the conservative approach (long-term, even very long-term antithyroid drug treatment) or the ablative approach (thyroidectomy, radioiodine, total thyroid ablation) can only be provided by large multicenter randomized clinical trials, not easy to perform.

\section{References}

1. L. Bartalena, A. Pinchera, C. Marcocci, Management of Graves' ophthalmopathy: reality and perspectives. Endocr. Rev. 21, 168-199 (2000)
2. L. Bartalena, M.L. Tanda, Clinical practice-Graves' ophthalmopathy. N. Engl. J. Med. 360, 994-1001 (2009)

3. L. Bartalena, L. Baldeschi, A. Dickinson, A. Eckstein, P. KendallTaylor, C. Marcocci, M. Mourits, P. Perros, K. Boboridis, A. Boschi, N. Currò, C. Daumerie, G.J. Kahaly, G.E. Krassas, C.M. Lane, J.H. Lazarus, M. Marinò, M. Nardi, C. Neoh, J. Orgiazzi, S. Pearce, A. Pinchera, S. Pitz, M. Salvi, P. Sivelli, M. Stahl, G. von Arx, W.M. Wiersinga, Consensus statement of the European Group on Graves' Orbitopathy (EUGOGO) on management of GO. Eur. J. Endocrinol. 158, 273-285 (2008)

4. S. Zang, K.A. Ponto, S. Pitz, G.J. Kahaly, Dose of intravenous steroids and therapy outcome in Graves' orbitopathy. J. Endocrinol. Invest. 34, 880-884 (2011)

5. L. Bartalena, What to do for moderate-to-severe and active Graves' orbitopathy if glucocorticoids fail? Clin. Endocrinol. (Oxf.) 73, 149-152 (2010)

6. T.J. Smith, Pathogenesis of Graves' orbitopathy: a 2010 update. J. Endocrinol. Invest. 33, 414-421 (2010)

7. L. Hegedus, T.J. Smith, R.S. Douglas, C.H. Nielsen, Targeted biological therapies for Graves' disease and thyroid-associated ophthalmopathy. Focus on B-cell depletion with Rituximab. Clin. Endocrinol. (Oxf.) 74, 1-8 (2011)

8. D. Paridaens, W.A. van den Bosch, T.L. van der Loos, E.P. Krenning, P.M. van Hagen, The effect of etanercept on Graves' ophthalmopathy. Eye 19, 1286-1289 (2005)

9. C. Marcocci, G.J. Kahaly, G.E. Krassas, L. Bartalena, M. Prummel, M. Stahl, M.A. Altea, M. Nardi, S. Pitz, K. Boboridis, P. Sivelli, G. von Arx, M.P. Mourits, L. Baldeschi, W. Bencivelli, W. Wiersinga, for the European Group on Graves' Orbitopathy, Selenium and the course of mild Graves' orbitopathy. N. Engl. J. Med. 364, 1920-1931 (2011)

10. C. Zhang, X. Zhang, L. Ma, F. Peng, J. Huang, H. Han, Thalidomide inhibits adipogenesis of orbital fibroblasts in Graves' ophthalmopathy. Endocrine 41 (2012). doi:10.1007/s12020-0129600-8

11. L. Bartalena, The dilemma of how to manage Graves' hyperthyroidism in patients with associated orbitopathy. J. Clin. Endocrinol. Metab. 96, 592-599 (2011)

12. M. Moleti, F. Mattina, I. Salamone, M.A. Violi, C. Nucera, S. Baldari, M.G. Lo Schiavo, C. Regalbuto, F. Trimarchi, F. Vermiglio, Effects of thyroidectomy alone or followed by radioiodine ablation of thyroid remnants on the outcome of Graves' ophthalmopathy. Thyroid 13, 653-658 (2003)

13. F. Menconi, M. Marinò, A. Pinchera, R. Rocchi, B. Mazzi, M. Nardi, L. Bartalena, C. Marcocci, Effects of total thyroid ablation versus near-total thyroidectomy alone on mild to moderate Graves' orbitopathy treated with intravenous glucocorticoids. J. Clin. Endocrinol. Metab. 92, 1653-1658 (2007)

14. A. De Bellis, G. Conzo, G. Cennamo, E. Pane, G. Bellastella, C. Colella, A. Dello Iacovo, V. Amoresano Paglionico, A.A. Sinisi, J.R. Wall, A. Bizzarro, A. Bellastella, Time course of Graves' ophthalmopathy after total thyroidectomy alone or followed by radioiodine therapy: a two-year longitudinal study. Endocrine 41 (2012). doi:10.1007/s12020-011-9559-x

15. L. Bartalena, C. Marcocci, A. Lai, M.L. Tanda, Graves' hyperthyroidism of recent onset and Graves' orbitopathy: to ablate or not to ablate the thyroid? J. Endocrinol. Invest. 31, 578-581 (2008) 